\title{
Splanchnic lipolysis in human obesity
}

\author{
Soren Nielsen, ${ }^{1}$ ZengKui Guo, ${ }^{1}$ C. Michael Johnson, ${ }^{2}$ Donald D. Hensrud, ${ }^{1}$ and Michael D. Jensen ${ }^{1}$
}

${ }^{1}$ Endocrine Research Unit and ${ }^{2}$ Department of Radiology, Mayo Clinic, Rochester, Minnesota, USA.

\begin{abstract}
Elevated FFA concentrations have been shown to reproduce some of the metabolic abnormalities of obesity. It has been hypothesized that visceral adipose tissue lipolysis releases excess FFAs into the portal vein, exposing the liver to higher FFA concentrations. We used isotope dilution/hepatic vein catheterization techniques to examine whether intra-abdominal fat contributes a greater portion of hepatic FFA delivery in visceral obesity. Obese women $(n=24)$ and men $(n=20)$ with a range of obesity phenotypes, taken together with healthy, lean women $(n=12)$ and men $(n=12)$, were studied. Systemic, splanchnic, and leg FFA kinetics were measured. The results showed that plasma FFA concentrations were approximately $20 \%$ greater in obese men and obese women. The contribution of splanchnic lipolysis to hepatic FFA delivery ranged from less than $10 \%$ to almost $50 \%$ and increased as a function of visceral fat in women $(r=0.49, P=0.002)$ and in men $(r=0.52, P=0.002)$; the slope of the relationship was greater in women than in men $(P<0.05)$. Leg and splanchnic tissues contributed a greater portion of systemic FFA release in obese men and women than in lean men and women. We conclude that the contribution of visceral adipose tissue lipolysis to hepatic FFA delivery increases with increasing visceral fat in humans and that this effect is greater in women than in men.
\end{abstract}

\section{Introduction}

A predominantly upper-body fat distribution is an important risk factor for the metabolic complications of obesity (1), especially when it is associated with increased intra-abdominal fat (2). Several metabolic abnormalities associated with upper-body obesity can be reproduced by excess FFAs, including insulin resistance with respect to muscle glucose uptake (3) and endogenous glucose production (4) and increased VLDL triglyceride production (5). Increased delivery of FFAs to the liver may be responsible for some of these abnormalities $(6,7)$. Visceral adipocytes are more lipolytically active than subcutaneous adipocytes in vitro $(8,9)$, suggesting that the association between greater amounts of visceral fat and the metabolic complications of obesity may reflect excess FFAs originating from visceral adipose tissue lipolysis (6, $7)$. These FFAs are released directly into the portal vein, exposing the liver to more FFAs than would be predicted from systemic FFA availability data. Thus, enlarged visceral fat stores could increase the proportion of hepatic FFA delivery coming from visceral, as opposed to systemic, sources.

The relationship between visceral fat and splanchnic FFA kinetics has not been assessed in humans. We previously reported a slight, but nonsignificant increase in splanchnic FFA (palmitate) release in upper-body obese women compared with lower-body obese and nonobese women (10). Visceral fat was not measured, however, and we did not include women with the full range of obesity-related metabolic abnormalities (10) in whom more significant disturbances of FFA metabolism might be expected. In addition, men were not studied, and obese men are more likely to preferentially gain visceral fat as they gain weight.

Because visceral fat is an important predictor of the metabolic complications of obesity, in this study we assessed splanchnic FFA

Nonstandard abbreviations used: free fatty mass (FFM); General Clinical Research Center (GCRC); resting energy expenditure (REE); specific activity (SA); waist-to-hip circumference ratio (WHR).

Conflict of interest: The authors have declared that no conflict of interest exists.

Citation for this article: J. Clin. Invest. 113:1582-1588 (2004).

doi:10.1172/JCI200421047. metabolism in individuals with a wide range of body fat, body fat distribution, and obesity-related health problems. A primary goal was to determine whether increased visceral fat is associated with proportionate increases in the fraction of FFAs reaching the liver directly from visceral adipose tissue lipolysis. We recently found that data collected using hepatic venous and arterial blood sampling provides good $\left(R^{2}=0.63\right)$ predictive value as to the proportion of hepatic FFA delivery that originates from visceral adipose tissue lipolysis (11). If the proportion of hepatic FFA delivery originating from visceral fat increases as visceral fatness increases, this would support the concept that one mechanism by which intraabdominal fat adversely affects health is by exposing the liver to more FFAs. If this were true, it would also indicate that measures of systemic FFA availability do not reflect the exposure of the liver to FFAs. As part of this study we also evaluated whether sex differences exist in systemic and regional FFA uptake and release as it relates to obesity.

\section{Methods}

Subjects. Experiments were approved by the Mayo Clinic Institutional Review Board prior to investigation, and informed, written consent was obtained from 12 nonobese men, 12 nonobese women, 20 obese men, and 24 obese women. All nonobese volunteers were in good health and taking no medications. The obese volunteers were selected to include a variety of phenotypes, including those with obesity-related health problems. Three obese women participants were hypertensive (diastolic blood pressures greater than 90 $\mathrm{mmHg}$ ), but were not being treated at the time of recruitment, and four obese men were hypertensive (two treated with medications). One obese man was treated with lipid-lowering agents, and one obese woman received insulin for glycemic management of type 2 diabetes. No other volunteers were taking antidiabetic medications, including metformin or thiazolidinediones. Volunteers were withdrawn from all antihypertensive and lipid-lowering agents for at least 1 month prior to the study, and those with diabetes were withdrawn from hypoglycemic therapy for at least 2 weeks prior to the study. All volunteers had maintained a stable weight for more than 2 months before the study and consumed all meals in the 
Mayo Clinic General Clinical Research Center (GCRC) for more than 3 days before the study to further ensure consistent energy and macronutrient intake. Because of the need to collect data from a large number of subjects, we modified several ongoing protocols to ensure that properly collected baseline data were available for analysis and included baseline data from a previous study of 18 obese women (12).

Materials. Indocyanine green (Cardio-Green; Becton Dickinson and Co., Cockeysville, Maryland, USA) was used for these studies. The FFA tracer used was $\left[9,10-{ }^{3} \mathrm{H}\right]$ palmitate (Amersham Life Sciences Inc., Arlington Heights, Illinois, USA), except for a subgroup of obese men and women in which we used $\left[1-{ }^{14} \mathrm{C}\right]$ palmitate (Amersham Life Sciences Inc.) in order to perform ancillary experiments regarding breath ${ }^{14} \mathrm{CO}_{2}$ excretion. We have previously shown that ${ }^{3} \mathrm{H}$ and ${ }^{14} \mathrm{C}$ FFA tracers provide the same FFA flux results (13).

Assays. The isotopic purity of the tracers, as well as the plasma palmitate, oleate, and total FFA concentrations and specific activity (SA) were determined using HPLC (14). Plasma insulin concentrations were measured using a two-site, $\mathrm{mAb}$ immuno-chemiluminometric assay (15). Plasma indocyanine green concentrations were measured on the day of the study using a spectrophotometer. Plasma glucose concentrations were measured using the glucose oxidase method, and plasma triglyceride concentrations were measured as previously described (16).

Protocol. Free fatty mass (FFM), total body fat, and leg fat were measured using dual-energy $\mathrm{x}$-ray absorptiometry (Lunar Radiation Corp., Madison, Wisconsin, USA) (17). Intra-abdominal fat and abdominal subcutaneous fat areas were measured at the $\mathrm{L}_{2-3}$ interspace using an Imatron C-100 (Imatron Inc., San Francisco, California, USA) computed tomography scanner (18).

Each subject was admitted to the Mayo Clinic GCRC the evening before the study, at which time an 18-gauge intravenous infusing catheter was placed in a forearm vein and kept patent with an infusion of $0.45 \% \mathrm{NaCl}$ at $30 \mathrm{ml} / \mathrm{h}$. Resting energy expenditure (REE) was measured by indirect calorimetry (DeltaTrac Metabolic Cart; SensorMedics Corp., Yorba Linda, California, USA) in the morning before the participants arose from bed. The metabolic cart was calibrated each morning prior to the study, and additional quality control for the carts included monthly pressure calibrations and gas calibrations together with biannual calibrations of the metabolic carts using an alcohol burn test.

After the indirect calorimetry, the participant was transferred to the Vascular Radiology Laboratory where a 5 French sheath was introduced into the right femoral artery using a standard percutaneous technique. A $20-\mathrm{cm}$-long 4 French straight catheter with six distally placed side holes was placed through the sheath with the catheter tip in the common iliac artery. This catheter was used for arterial blood sampling, and the sheath was used to infuse indocyanine green. The right femoral vein was then punctured in a similar manner, and a 6 French sheath was introduced. The distal tip of the sheath was placed in the external iliac vein a few centimeters above the inguinal ligament. A 5 French Simmons 2 catheter with four distal side holes was placed through the sheath, and the catheter tip was placed in the right hepatic vein. A solution of $0.45 \% \mathrm{NaCl}$ was infused through the sheaths and catheters to maintain patency. The volunteers were then transferred back to the GCRC for completion of the study. Blood was sampled before starting the isotope and the indocyanine green infusions to be used for background FFA SA and for construction of the indocyanine green calibration curve.
After returning to the GCRC, the volunteers rested in bed while infusions $-\left[9,10-{ }^{3} \mathrm{H}\right]$ palmitate or $\left[1-{ }^{14} \mathrm{C}\right]$ palmitate (approximately $0.3 \mu \mathrm{Ci} / \mathrm{min})$ and indocyanine green $(220 \mu \mathrm{g} / \mathrm{min})$ - were begun 30 minutes before blood sampling. The isotopes were infused through the forearm vein catheter, and the indocyanine green was infused into the femoral artery sheath to allow measurement of leg and splanchnic plasma flow $(19,20)$. Arterial, femoral venous, and hepatic venous blood samples were taken at 10 -minute intervals over a period of 30 minutes. After completion of the study, all catheters were removed, and local hemostasis was obtained. The subjects remained in the hospital under observation until the following morning.

Calculations. Systemic FFA rate of appearance and rate of disappearance $\left(R_{\mathrm{a}}\right.$ and $\left.R_{\mathrm{d}}\right)$ were calculated using the average arterial FFA palmitate SA and the tracer infusion rate corrected for isotopic purity (21). Steady-state plasma FFA concentrations and SA were used together with leg (19) and splanchnic (20) plasma flow to measure regional FFA uptake and release $(10,22)$. Upper-body nonsplanchnic FFA release is calculated as follows: Total FFA release $-[($ leg FFA release $\times 2)+$ splanchnic FFA release $]$.

The proportionate contribution of visceral lipolysis to hepatic FFA delivery was calculated using the values for splanchnic FFA release and splanchnic exit of arterial FFAs (11). Because leg FFA uptake is thought to occur largely in skeletal muscle, which is well predicted by leg FFM (23), leg FFA uptake is expressed relative to leg FFM.

The percentage of uptake of FFAs across the leg and splanchnic bed was calculated as follows: [(arterial FFA dpm $/ \mathrm{ml}$ - venous FFA $\mathrm{dpm} / \mathrm{ml}) \div$ arterial FFA $\mathrm{dpm} / \mathrm{ml}] \times 100 \%$

The model used to predict the percentage of hepatic FFA delivery from visceral adipose tissue lipolysis has been presented in detail (11). Simply stated, the basis of this model is that the SA (or enrichment in studies using stable isotopes) of FFAs appearing in the hepatic vein is equivalent to the average SA of FFAs delivered to the liver from the portal vein and hepatic artery relative to their respective plasma flows. Thus, the fractional reduction in hepatic vein FFA SA relative to the arterial FFA SA reflects the fraction of hepatic FFA delivery originating from visceral lipolysis. The salient assumptions of the model are as follows: (a) the tracer (in the case of these studies, ${ }^{3} \mathrm{H}$-palmitate) does not enter the bloodstream anywhere in the splanchnic bed, but only via the peripheral vein infusion; (b) new (unlabeled) FFAs do not enter the hepatic venous circulation other than from the portal vein; and (c) the efficiency of hepatic uptake of portally and arterially delivered FFA is essentially equal. The model was found to work well when plasma FFA concentrations were normal or high and less well when concentrations were suppressed (11).

Statistics. All results are expressed as mean plus or minus SEM. Comparisons among groups were made using ANOVA and subsequent two-tailed nonpaired $t$ test. To assess the relationship between body composition variables and regional FFA kinetics, linear regression analyses were performed. Statistical significance was taken as $P$ values less than 0.05 . Linear regression analysis was used to examine whether the relative contribution of visceral lipolysis to hepatic FFA delivery increased as visceral fat increased and to test whether visceral fat is associated with leg FFA uptake. To determine if there was a gender effect on the relationship between the percent of hepatic FFA delivery from visceral fat, this value was used as the dependent variable in a multivariate regression analysis using visceral fat area and sex as independent variables. A similar approach was used to examine the variables that relate to leg FFA uptake. 
Table 1

Volunteer characteristics

$\begin{array}{lcccc} & \text { Lean women } & \text { Obese women } & \text { Lean men } & \text { Obese men } \\ \text { Age (years) } & 30 \pm 1 & 37 \pm 1 & 31 \pm 2 & 40 \pm 1 \\ \text { Weight }(\mathrm{kg})^{\mathrm{B}} & 60.9 \pm 2.7 & 93.1 \pm 2.1 & 77.0 \pm 2.3 & 104.0 \pm 2.3 \\ \mathrm{BMI}\left(\mathrm{kg} / \mathrm{m}^{2}\right) & 21.6 \pm 0.7 & 33.8 \pm 0.5 & 23.6 \pm 0.5 & 32.9 \pm 0.7 \\ \text { Body fat }(\%)^{\mathrm{C}} & 29 \pm 2 & 48 \pm 1 & 19 \pm 1 & 32 \pm 1 \\ {\text { Leg fat }(\mathrm{kg})^{\mathrm{B}}}^{\mathrm{B}} & 7.1 \pm 0.6 & 15.5 \pm 0.8 & 4.8 \pm 0.5 & 10.6 \pm 0.7 \\ {\text { Leg lean }(\mathrm{kg})^{\mathrm{B}}}_{\text {Visceral fat area }\left(\mathrm{cm}^{2}\right)^{\mathrm{B}}}^{13.8 \pm 0.6} & 15.4 \pm 0.4 & 19.6 \pm 0.5 & 22.2 \pm 0.6 \\ \text { Abdominal subcutaneous } & 17 \pm 3 & 130 \pm 10 & 72 \pm 13 & 232 \pm 15 \\ \quad \text { fat area }\left(\mathrm{cm}^{2}\right)^{\mathrm{D}} & 79 \pm 14 & 339 \pm 22 & 91 \pm 14 & 266 \pm 17 \\ \text { Fasting serum insulin }(\mathrm{pmol} / \mathrm{l})^{\mathrm{A}} & 18 \pm 2 & 51 \pm 5 & 20 \pm 2 & 55 \pm 8 \\ \text { Fasting plasma glucose }(\mathrm{mmol} / \mathrm{l})^{\mathrm{E}} & 5.4 \pm 0.2 & 5.8 \pm 0.3 & 5.5 \pm 0.2 & 5.8 \pm 0.1 \\ \text { Serum triglycerides }(\mathrm{mg} / \mathrm{dl})^{\mathrm{A}} & 79 \pm 11 & 189 \pm 22 & 105 \pm 16 & 229 \pm 21 \\ & & & & \end{array}$

Values are mean \pm SEM. Lean women: $n=12$; obese women: $n=24$; lean men: $n=12$; obese men: $n=20$. ALean volunteers were significantly different from obese volunteers, but obese and lean did not differ between men and women. ${ }^{B}$ All groups significantly different. ${ }^{C}$ Only lean women and obese men did not differ. ${ }^{D}$ Only lean men and women did not differ. ENo between-group differences. BMI was a selection criteria and therefore not subject to statistical analysis.
The values for splanchnic uptake of arterially delivered FFAs and splanchnic FFA release are provided in Table 2. Splanchnic FFA uptake was not different between obese women and obese men, but was greater $(P<0.01)$ in both obese groups than in lean men and women. We examined whether the greater splanchnic uptake in obesity was solely a function of more delivery (FFA concentration times plasma flow) or if it was also related to greater fractional extraction of FFAs as they traverse this tissue bed. The fraction of systemic FFAs taken up across the splanchnic bed was $31 \% \pm 3 \%, 46 \% \pm 2 \%$, $36 \% \pm 4 \%$, and $44 \% \pm 2 \%$ in lean women, obese women, lean men, and obese men, respectively $(P<0.0001$ between group difference by ANOVA). The fractional uptake was less (at least $P<0.05$ ) in lean women than in all other groups, and fractional uptake in obese women was significantly greater than in lean men $(P=0.02)$.

\section{Results}

Subject characteristics. See Table 1. Although the mean BMI values were very similar in lean men and women and in obese men and obese women, women had more body fat $(P<0.001)$ than did men. The expected sex- and obesity-related differences in leg fat, visceral fat, and abdominal subcutaneous fat were present when comparing the lean and obese men and women. The mean waist-to-hip circumference ratio (WHR) for obese women was $0.86 \pm 0.02$ (range $0.74-1.00$ ) and for obese men was $0.99 \pm 0.01$ (range 0.93-1.09). The waist circumference of the obese women was $100 \pm 2 \mathrm{~cm}$ (range 88-113) and for the obese men was $109 \pm 2 \mathrm{~cm}$ (range 97-121). Fasting hyperinsulinemia and hypertriglyceridemia were present in obese men and women. The average fasting plasma glucose concentrations were greater in the obese subjects than in the lean subjects, although the difference did not reach statistical significance. The obese volunteers were an average of 7-9 years older than the nonobese volunteers $(P<0.05)$. REE was $1,547 \pm 61,1,791 \pm 60$, $1,714 \pm 46$, and $2,065 \pm 52 \mathrm{kcal} /$ day in lean women, lean men, obese women, and obese men, respectively.

Systemic FFA kinetics. Plasma FFA concentrations were $587 \pm 31$, $487 \pm 46,709 \pm 26$, and $663 \pm 38 \mu \mathrm{mol} / \mathrm{l}$ in lean women, lean men, obese women, and obese men, respectively (difference between groups, $P=0.002$ by ANOVA). Concentrations in obese men were greater than in lean men $(P=0.007)$ and lean women $(P=0.10)$, and concentrations in obese women were greater than in lean men $(P=0.0005)$ and lean women $(P=0.006)$. Within groups (obese and lean), men were not significantly different from women. We recently reported that REE is the best predictor of FFA flux and that FFA flux relates differently to REE in men and women (24). A similar relationship held true in this study group (data not shown), with women having a greater FFA relative to REE than did men. Total FFA flux in lean women, lean men, obese women, and obese men was $621 \pm 58,674 \pm 61,870 \pm 77$, and $678 \pm 44$ $\mu \mathrm{mol} / \mathrm{min}$, respectively.

Splanchnic FFA kinetics. Splanchnic plasma flow was not significantly different between lean women, lean men, obese women, and obese men (932 $\pm 43,871 \pm 68,963 \pm 71$, and $973 \pm 57 \mathrm{ml} / \mathrm{min}$, respectively).
There were no other significant between-group differences. This suggests that, at least in part, the greater splanchnic FFA uptake in obesity is related to greater efficiency of FFA extraction as they traverse splanchnic tissues.

Splanchnic FFA release was greater $(P<0.05)$ in both obese men and obese women than in lean men and women (Table 2), but not different between men and women within groups. The relative contribution of splanchnic FFA release to systemic FFA availability followed a similar pattern. For both lean women and men the splanchnic bed accounted for $6 \% \pm 1 \%$ of FFA release. In obese women splanchnic FFA release accounted for $13 \% \pm 2 \%(P<0.001$ versus both lean women and lean men) of systemic FFA release. In obese men it accounted for $17 \% \pm 2 \%(P<0.0005$ versus both lean women and lean men, $P=$ NS versus obese women) of systemic FFA release (Figure 1).

The percentage of FFAs delivered to the liver originating from visceral adipose tissue lipolysis was calculated for each participant as previously described (11). Visceral fat area was positively correlated with the percentage of hepatic FFA delivery from visceral fat

\section{Table 2}

\section{Regional FFA kinetics}

\begin{tabular}{lcccc} 
& Lean women & Lean men & Obese women & Obese men \\
Splanchnic & & & & \\
FFA uptake & $142 \pm 22$ & $150 \pm 20$ & $309 \pm 25^{\mathrm{B}}$ & $279 \pm 26^{\mathrm{B}}$ \\
FFA release & $35 \pm 8$ & $42 \pm 6$ & $111 \pm 16^{\mathrm{A}}$ & $111 \pm 16^{\mathrm{A}}$ \\
Leg & & & & \\
FFA uptake & $37 \pm 6$ & $38 \pm 5$ & $69 \pm 7^{\mathrm{B}}$ & $90 \pm 7^{\mathrm{B}}$ \\
FFA release & $61 \pm 7$ & $44 \pm 7$ & $107 \pm 10^{\mathrm{B}}$ & $107 \pm 11^{\mathrm{B}}$ \\
$\begin{array}{l}\text { Upper-body nonsplanchnic } \\
\text { FFA release }\end{array}$ & $462 \pm 46$ & $573 \pm 52$ & $544 \pm 70$ & $386 \pm 46$ \\
\hline
\end{tabular}

Values are micromoles per minute and expressed as mean \pm SEM. Leg FFA uptake and release values are for a single leg. Lean women: $n=12$; obese women: $n=24$; lean men: $n=12$; obese men: $n=20{ }^{A} P<0.05$ compared with lean women; $\mathrm{B} P<0.01$ compared with both lean groups. 


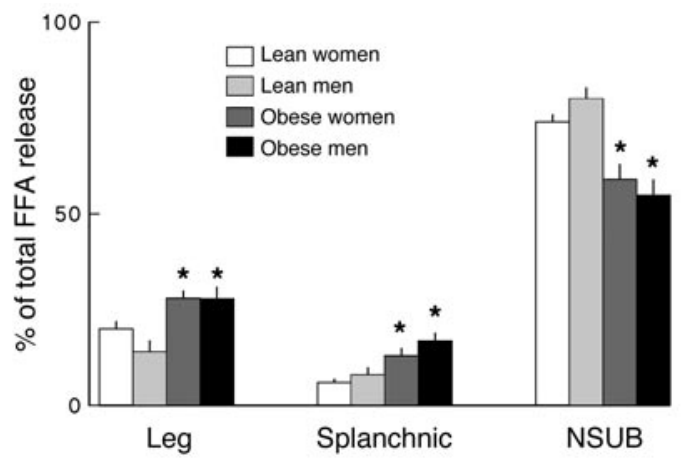

Figure 1

The percentage of systemic FFA release originating from leg, splanchnic, and nonsplanchnic upper body (NSUB) adipose tissue for lean and obese men and women are plotted. ${ }^{\star} P<0.05$ vs. lean.

both in women $(r=0.49, P=0.002$; Figure 2$)$ and in men $(r=0.52$, $P=0.002$; Figure 2). Note the relationships do not appear to be identical in men and women. To test whether the difference was significant we used multivariate regression analysis with the percentage of hepatic FFA delivery from visceral fat as the dependent variable and visceral fat area and sex as independent variables. Both visceral fat area $(P=0.0001)$ and sex $(P=0.04)$ were significant predictors of visceral fat FFA delivery to the liver.

Leg FFA kinetics. Leg plasma flow was not significantly different between lean women and men and obese women and men $(315 \pm 31$, $242 \pm 27,319 \pm 20$, and $317 \pm 29 \mathrm{ml} / \mathrm{min}$, respectively).

Leg FFA uptake and release values (micromoles per minute) are provided in Table 2. Both leg FFA uptake and release were greater $(P<0.01)$ in obese participants than in lean participants, but not different between men and women in their respective obese and lean groups. In addition, the relative contribution of lower-body adipose tissue lipolysis (both legs) to systemic FFA release was different among groups $(P=0.02$, ANOVA). In lean women and men the lower body contributed $20 \% \pm 2 \%$ and $14 \% \pm 3 \%$ of systemic FFAs, whereas in obese men and women the contribution was $28 \% \pm 3 \%$ and $28 \% \pm 2 \%$, respectively (Figure 2 ).

Because of previously reported negative associations between visceral fat and leg FFA uptake (25), we examined the relationship between variables known to be associated with leg FFA uptake. As expected $(26,27)$, leg FFA uptake was positively correlated with plasma FFA concentrations in men and women (Figure 3A). To test for other factors related to leg FFA uptake multivariate regression analysis was used. Independent variables included in the model were plasma FFA concentration, sex, and visceral fat area. In this model both FFA concentrations $(P=0.004)$ and visceral fat area $(P=0.02)$ were independent predictors of leg FFA uptake, but sex was not. Of note, the parameter estimate for visceral fat was positive $(0.017 \pm 0.007)$, indicating that greater visceral fat was predictive of greater leg FFA uptake after accounting for plasma FFA concentrations. To visualize this interaction we plotted the residual variance between plasma FFA concentrations and leg FFA uptake as a function of visceral fat area (Figure 3, right panel). The residual variance in leg FFA uptake is calculated by subtracting the predicted leg FFA uptake - based on plasma FFA concentration regression formulas - from the observed leg FFA uptake for each subject. Consistent with the multivariate analysis, there was a significant correlation between visceral fat and the residual variance in leg FFA uptake $(r=0.32, P=0.008)$.
Leg FFA uptake was divided by total FFA flux to determine what proportion of systemic FFAs is removed by the leg. Leg tissue accounted for $13 \% \pm 2 \%$ and $10 \% \pm 1 \%$ of systemic FFA removal in lean women and lean men, respectively, but took up $17 \% \pm 2 \%$ and $22 \% \pm 2 \%$ of total FFA uptake in obese women and obese men (group difference by ANOVA $=0.0008$ ). Lean men and women were not different, and obese men and women were not different. The difference between lean and obese women was not statistically significantly $(P=0.096)$. Thus, the trend was for a greater proportion of systemic FFA disposal to be in the leg tissue of obese adults.

To determine whether this was due primarily to a more efficient removal process, the fraction of FFAs taken up across the leg was calculated. Using the fractional tracer uptake data we determined that $22 \% \pm 2 \%, 30 \% \pm 3 \%, 41 \% \pm 4 \%$, and $36 \% \pm 3 \%$ of FFAs were taken up during transit through leg tissue in lean women, obese women, lean men, and obese men, respectively $(P<0.005$ between group difference by ANOVA). The fractional uptake was less (at least $P<0.03)$ in lean women than all other groups, and fractional uptake in obese women was less $(P=0.04)$ than in lean men. There were no other significant between-group differences. These values suggest that the differences in plasma FFA concentration, rather than efficiency of uptake, is the main explanation for the greater leg FFA uptake in obesity

Upper-body nonsplanchnic FFA release. Upper-body nonsplanchnic FFA release values (micromoles per minute) are provided in Table 2. When compared on this basis there were no significant differences among groups. When this tissue bed's contribution to systemic FFA release was examined as a percentage of total FFA release, substantial between-group differences were evident $(P=0.001$ by ANOVA; see Figure 1$)$. Although upper-body nonsplanchnic FFA release was the major contributor to systemic FFA availability in obese adults, it provided a lesser fraction than it did in nonobese adults.

\section{Discussion}

On the basis of our previous studies of regional FFA metabolism $(10,12,28-31)$ we have emphasized the importance of upper-body nonsplanchnic fat as the major contributor to systemic FFA availability. In this study we addressed an issue we could not deal with in

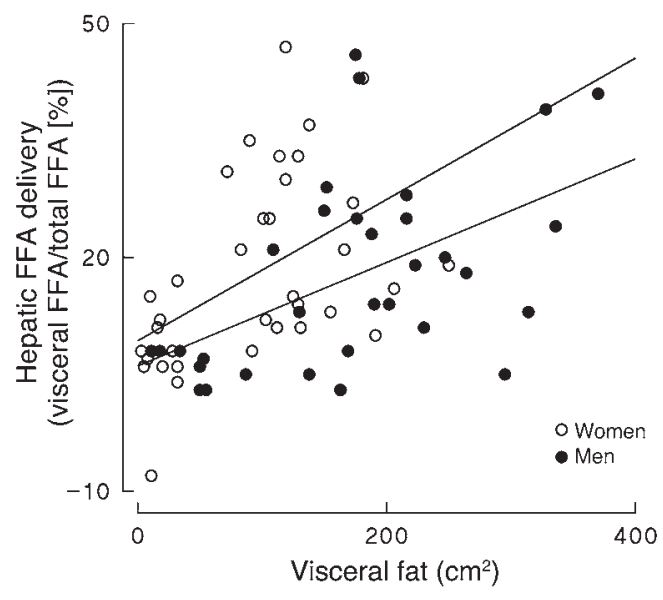

\section{Figure 2}

The percentage of hepatic FFA delivery originating from visceral adipose tissue lipolysis for men and women participants of the study are plotted versus visceral fat area. 


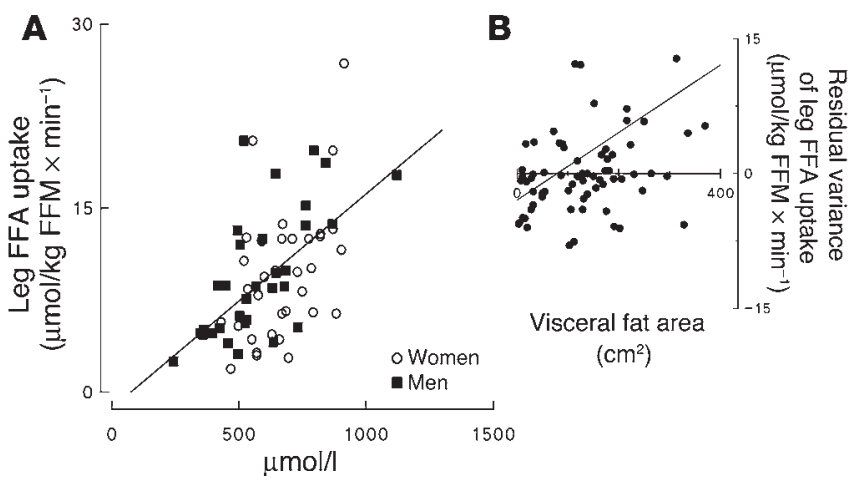

Figure 3

Leg FFA uptake is plotted versus plasma FFA concentration for women and men participating in the study (A). The residual variance of leg FFA uptake is plotted versus visceral fat area for all study participants (B).

the previous studies: does visceral fat play a special role in determining hepatic FFA delivery? It has been postulated that visceral adipose tissue has a direct adverse effect on human health by releasing excess FFAs into the portal vein, thereby exposing the liver of viscerally obese persons to greater FFA concentrations (7). Because accessing the portal vein to test this hypothesis is impractical for human studies, we developed and validated a model that uses hepatic vein blood samples to predict the relative contribution of visceral adipose tissue lipolysis to hepatic FFA delivery (11). This study employed that model to help understand whether visceral lipolysis contributes relatively more FFAs to the liver in those with greater visceral fat and whether women differ from men in this regard. We recruited volunteers with a wide range of obesity phenotypes to ensure those with obesity-related health complications would be represented. This study provides, we believe for the first time, in vivo evidence that a greater portion of hepatic FFA delivery originates from visceral adipose tissue lipolysis in individuals with more visceral fat. We also found that this relationship is more accentuated in women than in men. These findings have implications for understanding the regulation of hepatic glucose and lipoprotein metabolism.

Increased delivery of FFAs to the liver is thought to result in increased VLDL triglyceride production $(5,32)$ and insulin resistance with regard to hepatic glucose production (4). The positive association between visceral fat and the metabolic complications of obesity has been attributed to high rates of visceral adipose tissue FFA release into the portal vein, resulting in greater hepatic FFA delivery $(6,7)$. If this explanation is correct, a greater proportion of FFAs reaching the liver must come from visceral fat in this form of obesity. We found that in adults with lesser amounts of visceral fat, as little as $5-10 \%$ of hepatic FFA delivery originates from visceral adipose tissue lipolysis. In contrast, in some individuals with greater amounts of visceral fat, over $30 \%$ of hepatic FFA delivery came from visceral lipolysis. The remainder of FFAs reaching the liver are those entering the splanchnic bed from the systemic circulation that escape uptake by nonhepatic splanchnic tissues. If this nonhepatic FFA uptake process is not greater in viscerally obese than in lean persons, post-absorptive hepatic FFA delivery could be more than $20 \%$ greater in visceral obesity. We should point out that even in viscerally obese adults FFAs delivered to the splanchnic bed from the systemic circulation generally provides more than $50-60 \%$ of hepatic FFA delivery.
We used a model (11) to predict the percent of FFAs delivered to the liver from visceral lipolysis because a direct measure would require portal vein sampling, a procedure not applicable to human studies. In our validation study of this model the correlation between model predicted values and measured values was approximately 0.79 (11). Because of this imperfect relationship, a relatively large number of volunteers were included in each group to ensure the statistical power to detect a modest association between visceral fat and visceral fat FFA delivery to the liver. We also included volunteers with a wide range of fat and fat distribution to ensure that if a relationship between visceral adiposity and hepatic FFA delivery is present, we could detect it. It is possible that the relationship between visceral adiposity and the relative contribution of visceral lipolysis to hepatic FFA delivery is better than we describe herein. Establishing that there is a relationship and identifying the relative contribution of visceral lipolysis to hepatic FFA delivery provides a better understanding of the physiology. In addition, the finding that the contribution of visceral lipolysis to hepatic FFA delivery is greater in relation to visceral fat in women than in men is a novel finding that could have broader implications. For example, it has been reported that the correlation between visceral adipose tissue area and serum triglycerides is better in women than in men (33). If hepatic FFA delivery drives hepatic VLDL production this would be consistent with our observation. The hepatic insulin resistance index (as regards glucose metabolism) was significantly correlated with visceral fat in women, but not in men (34). Considering the role of FFAs in modulating hepatic glucose production (35), our findings may provide a physiological explanation for this observed sex difference (34) in the relationships.

One of the limitations of the model we employ to estimate the proportion of hepatic FFA delivery originating from visceral adipose tissue lipolysis is that if FFAs are released directly into the hepatic venous circulation, they would be attributed to visceral lipolysis (11). In our validation studies we observed more model error at low plasma FFA concentrations/flux (11). One of the explanations we considered is a limited release of FFAs into the hepatic venous effluent. This could be from incomplete hepatic uptake of fatty acids released from triglyceride-rich lipoproteins by the action of hepatic lipase or from hepatocyte release of fatty acids after intracellular hydrolysis of fatty acid-containing compounds. Of these two possible explanations we favor the former because the adult liver does not contain the enzymatic/ protein machinery (hormone-sensitive lipase perilipin-A, or aP2) to behave as a competent lipolytic organ. In any case, our previous results (11) suggest that the potential error would be quantitatively important only in low FFA flux environments, which is not a concern in the present study. This conclusion is consistent with the thoughts of Havel et al. (36), who performed some of the first and most elegant studies of hepatic/splanchnic FFA metabolism in humans. These investigators provided novel information regarding the hepatic use of FFA for VLDL triglyceride production, ketone body production, and oxidation (36). Based upon their animal studies (37), they concluded there was little likelihood of FFA release in the hepatic circulation per se, and they estimated that $80 \%$ of splanchnic FFA uptake occurred in the liver. The $80 \%$ figure was used to calculate total hepatic FFA uptake in their human studies (36). We took a somewhat different approach, using individual differences in hepatic vein FFA SA to understand visceral adipose tissue lipolysis; visceral adiposity was not an issue at the time of Havel's studies (36). 
Leg FFA uptake strongly correlated with plasma FFA concentrations, but as assessed by multiple regression analysis was additionally impacted by visceral fatness. Those with greater amounts of visceral fat had greater leg FFA uptake. This is in contrast to the findings of Colberg et al. (25), who found a negative relationship between leg FFA uptake and visceral fat mass. It is difficult to make direct comparisons between the two studies because some of the data we found to be important in evaluating our findings (leg tissue composition, individual plasma FFA concentrations) were either not available or not reported by Colberg et al. (25). For example, plasma FFA concentrations are a predictor of FFA uptake, yet this was not included as a variable in the analysis by Colberg et al. (25). Our statistical approach used FFA concentration as an independent variable and assessed whether visceral fat has additive, independent predictive value. In addition, because the measures of leg FFM were not available to Colberg et al. (25), leg FFA uptake data were analyzed as $\mathrm{nmol} / \mathrm{min}$ (as opposed to $\mu \mathrm{mol} / \mathrm{kg} \mathrm{FFM} / \mathrm{min}$ as we were able to do). This could be misleading if some women had larger amounts of muscle, which would be expected to take up more FFAs. There was no relationship between visceral adiposity and plasma FFA concentrations for the 17 lean and obese women (25) studied, which is unexpected based upon the literature and different from our results, where we found significant, positive relationships between visceral adiposity and plasma FFA concentrations in both men and women. This suggests that subject selection factors may have contributed to the discrepant findings. Finally, the conclusion that visceral fat is inversely related to leg FFA uptake rests primarily on a steep decline in uptake in individuals with visceral fat areas between 0 and $80 \mathrm{~cm}^{2}$; no such relationship is apparent in women with visceral fat areas $80-180 \mathrm{~cm}^{2}(25)$. Because we studied more subjects, covaried for plasma FFA concentrations while testing for the visceral fat effect, included a wider range of visceral adiposity, and accounted for leg FFM, we believe our findings are more likely to be representative of the pathophysiology of obesity. Colberg et al. (25) may have been the victim of unintended subject selection bias or a type 1 statistical error centered on those with visceral fat areas less than $80 \mathrm{~cm}^{2}$. For the results of both studies it should be noted that $10-20 \%$ of leg blood flow is probably directed toward adipose tissue rather than muscle. Although adipose tissue does not appear to take up substantial amounts of FFAs in the postabsorptive state (38), we cannot exclude the possibility that a fraction of the leg FFA uptake we observed was into adipose tissue, not skeletal muscle. Thus, the leg uptake values we report (in units of micromoles $\times \mathrm{kg}$ leg $\mathrm{FFM}^{-1} \times \mathrm{min}^{-1}$ ) may be slight overestimates of leg muscle FFA uptake.

Fasting plasma FFA concentrations were approximately $20 \%$ greater in the obese volunteers than in the lean volunteers. The higher plasma FFA concentrations in obese women were accompanied by approximately $40 \%$ greater FFA release, which is in contrast to men, where the $20 \%$ greater plasma FFA concentrations in obese men was in the face of virtually identical FFA release rates. Although we considered the possibility that reduced clearance rather than increased lipolysis accounted for the higher plasma FFA concentrations in men, other results indicate this is unlikely. Data from a previous study that included lean and obese men (24) was reanalyzed, dividing men into those with a BMI above $25(n=13$, average BMI $=30.2$ $\left.\mathrm{kg} / \mathrm{m}^{2}\right)$ and below $25\left(n=12\right.$, average BMI $\left.=23.2 \mathrm{~kg} / \mathrm{m}^{2}\right)$. The overweight men had $20 \%$ greater fasting plasma palmitate concentrations (92 \pm 5 versus $76 \pm 5 \mu \mathrm{mol} / \mathrm{l}), P=0.015)$, approximately $20 \%$ greater palmitate rate of appearance $(94 \pm 5$ versus $78 \pm 8 \mu \mathrm{mol} / \mathrm{min}$, $P=0.051)$, and $7 \%$ greater REE $(1,941 \pm 46$ versus $1,815 \pm 67 \mathrm{kcal} /$ day, $P=0.06)$. The increase in FFA appearance out of proportion to REE in this group (24) implies greater lipolysis in obese men and does not support the conclusion that reduced FFA clearance generally accounts for greater plasma FFA concentrations.

In summary, these studies have assessed the relative contribution of visceral fat to hepatic FFA delivery in lean and obese men and women. In both men and women, visceral adipose tissue lipolysis accounts for an increasing proportion of hepatic FFA delivery as visceral fat increases; the slope of the relationship seems to be steeper in women than in men. This is strong evidence that in the post-absorptive state the liver of viscerally obese humans is exposed to higher concentrations of FFAs. Thus, visceral adiposity relates to abnormal FFA metabolism in (at least) two ways: excess release of FFAs from upper body subcutaneous fat $(10,12,30)$ and increased hepatic FFA delivery. This also indicates that measuring arterial plasma FFA concentrations does not reflect hepatic FFA delivery in viscerally obese humans. We also found that leg FFA uptake is primarily a function of plasma FFA concentrations and is increased in visceral obesity.

\section{Acknowledgments}

This work was supported by grants from the US Public Health Service (DK-40484, DK-45343, and DK-50456, to M.D. Jensen), and from the Mayo Foundation (RR-0585). We acknowledge the technical assistance of Carol Siverling and Darlene Lucas, as well the staff of the Mayo Clinic GCRC and the Mayo Clinic Vascular Radiology Department. We acknowledge Monica Davis for her editorial assistance.

Received for publication January 13, 2004, and accepted in revised form March 23, 2004.

Address correspondence to: Michael D. Jensen, Endocrine Research Unit, 5-164 West Joseph, Mayo Clinic, Rochester, Minnesota 55095, USA. Phone: (507) 255-6768; Fax (507) 255-4828; E-mail: jensen.michael@mayo.edu.
1. Kissebah, A.H., et al. 1982. Relation of body fat distribution to metabolic complications of obesity. J. Clin. Endocrinol. Metab. 54:254-260.

2. Fujioka, S., Matsuzawa, Y., Tokunaga, K., and Tarui, S. 1987. Contribution of intra-abdominal fat accumulation to the impairment of glucose and lipid metabolism in human obesity. Metabolism. 36:54-59.

3. Kelley, D.E., Mokan, M., Simoneau, J.A., and Mandarino, L.J. 1993. Interaction between glucose and free fatty acid metabolism in human skeletal muscle. J. Clin. Invest. 92:91-98.

4. Ferrannini, E., Barrett, E.J., Bevilacqva, S., and
DeFronzo, R.A. 1983. Effect of fatty acids on glucose production and utilization in man. J. Clin. Invest. 72:1737-1747.

5. Lewis, G.F., Uffelman, K.D., Szeto, L.W., Weller, B., and Steiner, G. 1995. Interaction between free fatty acids and insulin in the acute control of very low density lipoprotein production in humans. J. Clin. Invest. 95:158-166.

6. Kissebah, A.H., and Peiris, A.N. 1989. Biology of regional body fat distribution: relationship to non-insulin-dependent diabetes mellitus. Diabetes Metab. Rev. 5:83-109.

7. Bjorntorp, P. 1990. "Portal” adipose tissue as a gen- erator of risk factors for cardiovascular disease and diabetes. Arteriosclerosis. 10:493-496.

8. Rebuffe-Scrive, M., Andersson, B., Olbe, L., and Bjorntorp, P. 1989. Metabolism of adipose tissue in intra-abdominal depots of nonobese men and women. Metabolism. 38:453-458.

9. Mauriege, P., Galitzky, J., Berlan, M., and Lafontan, M. 1987. Heterogeneous distribution of beta and alpha-2 adrenoceptor binding sites in human fat cells from various fat deposits: functional consequences. Eur. J. Clin. Invest. 17:156-165.

10. Martin, M.L., and Jensen, M.D. 1991. Effects of body fat distribution on regional lipolysis in obe- 
sity. J. Clin. Invest. 88:609-613.

11. Jensen, M.D., Cardin, S., Edgerton, D., and Cherrington, A. 2003. Splanchnic free fatty acid kinetics. Am. J. Physiol. Endocrinol. Metab. 284:E1140-E1148.

12. Guo, Z.K., Hensrud, D.D., Johnson, C.M., and Jensen, M.D. 1999. Regional postprandial fatty acid metabolism in different obesity phenotypes. Diabetes. 48:1586-1592.

13. Heiling, V.J., Miles, J.M., and Jensen, M.D. 1991. How valid are isotopic measurements of fatty acid oxidation? Am. J. Physiol. 261:E572-E577.

14. Miles, J.M., Ellman, M.G., McClean, K.L., and Jensen, M.D. 1987. Validation of a new method for determination of free fatty acid turnover. Am. J. Physiol. Endocrinol. Metab. 252:E431-E438.

15. Klee, G., Furlow, B., Shellum, C., and Smith, T. 1995. An automated immuno-chemiluminometric insulin assay for the access immunoassay system. Clin. Chem. 41:S34 (Abstr.)

16. Humphreys, S.M., Fisher, R.M., and Frayn, K.N. 1990. Micromethod for measurement of sub-nanomole amounts of triacylglycerol. Ann. Clin. Biochem. 27:597-598.

17. Jensen, M.D., et al. 1993. Assessment of body composition with use of dual-energy $\mathrm{x}$-ray absorptiometry: evaluation and comparison with other methods. Mayo Clin. Proc. 68:867-873.

18. Jensen, M.D., Kanaley, J.A., Reed, J.E., and Sheedy, P.F. 1995. Measurement of abdominal and visceral fat with computed tomography and dual-energy x-ray absorptiometry. Am. J. Clin. Nutr. 61:274-278.

19. Jorfeldt, L., and Wahren, J. 1971. Leg blood flow during exercise in man. Clin. Sci. 41:459-473.
20. Bradley, S.E., Ingelfinger, F.J., Bradley, G.P., and Curry, J.J. 1945. The estimation of hepatic blood flow in man. J. Clin. Invest. 24:890-897.

21. Guo, Z., Johnson, C.M., and Jensen, M.D. 1997. Regional lipolytic responses to isoproterenol in women. Am. J. Physiol. 273:E108-E112.

22. Jensen, M.D. 1991. Regulation of forearm lipolysis in different types of obesity. In vivo evidence for adipocyte heterogeneity. J. Clin. Invest. 87:187-193.

23. Levine, J.A., et al. 2000. Measuring leg muscle and fat mass in humans. J. Appl. Physiol. 88:452-456

24. Nielsen, S., et al. 2003. Energy expenditure, sex, and endogenous fuel availability in humans. J. Clin. Invest. 111:981-988. doi:10.1172/JCI200316253.

25. Colberg, S., Simoneau, J.A., Thaete, F., and Kelley, D.E. 1995. Skeletal muscle utilization of free fatty acids in women with visceral obesity. J. Clin. Invest. 95:1846-1853.

26. Spitzer,J.J., and Gold, M. 1964. Free fatty acid metabolism by skeletal muscle. Am. J. Physiol. 206:159-163.

27. Turcotte, L.P., Kiens, B., and Richter, E.A. 1991. Saturation kinetics of palmitate uptake in perfused skeletal muscle. FEBS Lett. 279:327-329.

28. Jensen, M.D. 1995. Gender differences in regional fatty acid metabolism before and after meal ingestion. J. Clin. Invest. 96:2297-2303.

29. Jensen, M.D., and Johnson, C.M. 1996. Contribution of leg and splanchnic free fatty acid (FFA) kinetics to postabsorptive FFA flux in men and women. Metabolism. 45:662-666.

30. Basu, A., et al. 2001. Systemic and regional free fatty acid metabolism in type 2 diabetes. Am. J. Physiol. Endocrinol. Metab. 280:E1000-E1006.
31. Meek, S., Nair, K.S., and Jensen, M.D. 1999. Insulin regulation of regional free fatty acid metabolism. Diabetes. 48:10-14.

32. Kissebah, A.H., Alfarsi, S., Adams, P.W., and Wynn, V. 1976. Role of insulin resistance in adipose tissue and liver in the pathogenesis of endogenous hypertriglyceridaemia in man. Diabetologia. 12:563-571.

33. Smith, S.R., et al. 2001. Contributions of total body fat, abdominal subcutaneous adipose tissue compartments, and visceral adipose tissue to the metabolic complications of obesity. Metabolism. 50:425-435.

34. Miyazaki, Y., et al. 2002. Abdominal fat distribution and peripheral and hepatic insulin resistance in type 2 diabetes mellitus. Am. J. Physiol. Endocrinol. Metab. 283:E1135-E1143.

35. Rebrin, K., Steil, G.M., Getty, L., and Bergman, R.N. 1995. Free fatty acids as a link in the regulation of hepatic glucose output by peripheral insulin. Diabetes. 44:1038-1045.

36. Havel, R.J., Kane, J.P., Balasse, E.O., Segel, N., and Basso, L.V. 1970. Splanchnic metabolism of free fatty acids and production of triglycerides of very low density lipoproteins in normotriglyceridemic and hypertriglyceridemic humans. J. Clin. Invest. 49:2017-2035.

37. Basso, L.V., and Havel, R.J. 1970. Hepatic metabolism of free fatty acids in normal and diabetic dogs. J. Clin. Invest. 49:537-547.

38. Coppack, S.W., Persson, M., Judd, R.L., and Miles, J.M. 1999. Glycerol and nonesterified fatty acid metabolism in human muscle and adipose tissue in vivo. Am. J. Physiol. 276:E233-E240. 